\title{
Separation and Determination of Yellow Gardenia Pigments for Food and Iridoid Constituents in Gardenia Fruits by Micellar Electrokinetic Chromatography
}

\author{
Toshiro Watanabe, ${ }^{1}$ Akira Yamamoto, ${ }^{1}$ Shiro Nagai ${ }^{1}$ and Shigeru Terabe ${ }^{2}$ \\ ${ }^{1}$ Yaegaki Technology Development Laboratories, Yaegaki Bio-industry, Inc., 681 Hayashida, Himeji, Hyogo 679-42, Japan \\ ${ }^{2}$ Faculty of Science, Himeji Institute of Technology, Kamigori, Hyogo 678-12, Japan
}

Received June 20, 1997; Accepted September 20, 1997

\begin{abstract}
Micellar electrokinetic chromatography (MEKC) methods were developed for analyzing crocin and crocetin yellow gardenia pigments, and geniposide and gardenoside in gardenia fruits. Crocin and crocetin pigments were successfully separated by MEKC with a $20 \mathrm{mM}$ SDS solution in $50 \mathrm{mM}$ phosphate buffer (pH 7.0) containing $20 \%$ acetonitrile and another MEKC method with a $20 \mathrm{mM}$ ammonium formate buffer (pH 7.0) containing $2.0 \%$ butyl acrylate/butyl methacrylate/methacrylic acid copolymer sodium salts (BBMA). Geniposide and gardenoside were also successfully separated by MEKC with a 20 mM sodium dodecyl sulfate (SDS) solution in 30 mM borate buffer (pH 8.5). The crocin and crocetin yellow pigments were extracted from food samples (candies and noodles) by solid-phase extraction cartridges and analyzed by MEKC with SDS. The geniposide and gardenoside in gardenia fruits grown in different habitats were determined by the developed technique.
\end{abstract}

Keywords: micellar electrokinetic chromatography, crocin, crocetin, geniposide, gardenoside

The constituents of gardenia fruits ( Gardenia jasminoides ELLIS) are known as herb medicine and natural dye in China. The fruits produce yellow carotenoid pigments and iridoid compounds. The two main components in the yellow pigments are called crocin and crocetin (Fig. 1) (Ichi et al., 1995a, b, c), and the yellow pigments have been used as a natural food colorant for a long time in Japan, mainly in colored juice, jello, candy and noodles, because of their water solubility. The iridoid constituents of gardenia fruits, which are used as tranquilizers, are called geniposide and gardenoside as shown in Fig. 1 (Touyama et al., 1994). In recent years, natural food additives have drawn renewed attention from the viewpoint of safety, and an analytical method for natural food additives is thus important. However, analyzing food additives extracted from food is very difficult because of their extremely low concentrations (Ogiwara et al., 1980; Nishizawa et al., 1983).

Micellar electrokinetic chromatography (MEKC) was developed by Terabe et al. (1984), and they referred to the technique as electrokinetic separations. In a later paper (Terabe et al., 1985), it was termed electrokinetic chromatography because it was considered that it is a type of chromatography similar to liquid-liquid partition chromatography. This mode of capillary electrophoresis (CE) is now commonly referred to as micellar electrokinetic chromatography; it is abbreviated as MEKC to comply with the term micellar EKC as used by Nishi et al. (1989). MEKC, in which an ionic micellar solution is employed as the running solution, is a popular separation mode for $\mathrm{CE}$ when small molecules are involved. With MEKC, electrically neutral analytes can be separated by their different ratio of partitioning to the micelle.

Although there are a few reports on the analysis of yellow gardenia pigments (Kamikura \& Nakazato, 1985) and iridoid gardenia constituents (Noda et al, 1983; Kamikura \& Nakazato, 1984) by high-performance liquid chromatography (HPLC), to the best of our knowledge, there are no analytical reports using CE, except for those by us (Watanabe et al., 1996; 1997a, b). This paper describes the analysis of yellow gardenia pigments, the extraction of iridoid constituents from gardenia fruits and their analysis by CE.

\section{Materials and Methods}

Materials Two kinds of commercial yellow gardenia pigments for food, one (pigment A) containing mainly crocin and the other (pigment B) mainly crocetin, are produced by Yaegaki Bio-industry (Himeji). Dried gardenia fruits were purchased from China, Taiwan and Korea. Geniposide and gardenoside were purchased from Wako Pure Chemicals (Osaka). Sodium dodecyl sulfate (SDS) from Nacalai Tesque (Kyoto) and butyl acrylate / butyl methacrylate / methacrylic acid copolymer sodium salts (BBMA) from Daiichi Kogyo Seiyaku (Kyoto) were used as anionic micellar pseudostationary phases for MEKC. Pure water was prepared by purifying distilled water with a Milli-Q SP system (Millipore, Bedford, MA) just before use. All other chemicals and solvents were of analytical reagent grade.

Apparatus CE was performed with a BioFocus 3000 CE system (Bio-Rad, Richmond, CA), using a pre-packed cartridge of an uncoated fused silica capillary of $50 \mu \mathrm{m}$ i.d. and a total length of $50 \mathrm{~cm}$ ( $46 \mathrm{~cm}$ to the detector). HPLC separation and purification were performed by a Beckman Gold HPLC system with a programmable 125 solvent module and programmable 166 detector module (Fullerton, CA). For analytical separation, an Inertsil ODS-2 packed column (4.6 mm i.d. and $150 \mathrm{~mm}$ length with a $5-\mu \mathrm{m}$ particle size; GL Science, Tokyo) was used, and a Shim-pack 


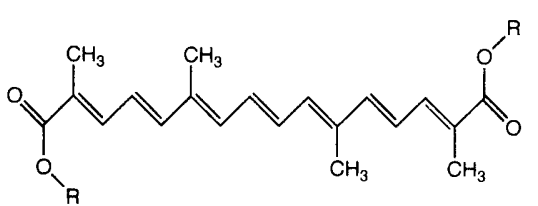

Crocin : $\mathbf{R}=$ Gentiobiose Crocetin : R=H<smiles>[R]OC1OC=C(C(C)=O)C2C=CC([R])(CO)C12</smiles>

Gardenoside : $\mathrm{R}=\mathrm{OH}$ Geniposide : $\mathbf{R}=\mathbf{H}$

Fig. 1. Structures of crocin, crocetin, geniposide and gardenoside.

PREP-ODS packed column $(20.0 \mathrm{~mm}$ i.d. and $250 \mathrm{~mm}$ length; Shimadzu, Kyoto) was used for preparative separation.

Extraction of the geniposide and gardenoside from gardenia fruits Gardenia fruit was shattered by a smallsized crush machine. The shattered gardenia fruit $(0.1 \mathrm{~g})$ was extracted with $100 \mathrm{ml}$ of distilled water for $60 \mathrm{~min}$. The extracts were passed through a filter paper $(110 \mathrm{~mm}$, No. 2; Advantec Toyo, Tokyo), diluted and analyzed for geniposide and gardenoside contents using HPLC and CE.

Extraction of yellow pigments from food samples Yellow pigments containing crocin and crocetin (pigments $A$ and B) were extracted by a solid-phase extraction (SPE) method from candies and noodles. Candies were dissolved in warm distilled water $\left(75^{\circ} \mathrm{C}\right)$ and noodles were extracted in methanol solution (methanol: distilled water $=1: 1$ ). These pigment solutions were evaporated to dryness at $35^{\circ} \mathrm{C}$, and the residues were dissolved in water before the SPE method. Prior to use, the SPE cartridge (Bond Elut, $500 \mathrm{mg} / 6 \mathrm{ml}$; Varian, Harbor, CA) was pre-conditioned with $10 \mathrm{ml}$ of methanol and then with $20 \mathrm{ml}$ of distilled water. Each pigment solution $(10 \mathrm{ml})$ was applied to the SPE cartridge, and the SPE cartridge was washed with $20 \mathrm{ml}$ of distilled water. Yellow gardenia pigment was eluted with $5 \mathrm{ml}$ of methanol. Eluants were evaporated to dryness at $35^{\circ} \mathrm{C}$. The residue was dissolved in water and subjected to the MEKC analysis.

HPLC separation and preparation Gradient elution was performed for both analytical and preparative separations of yellow pigments with distilled water containing $0.05 \%$ trifluoroacetic acid (TFA) (sol. A, $\mathrm{pH} \mathrm{2.5)}$ ) and with $100 \%$ acetonitrile containing $0.05 \%$ TFA (sol. B). A lineargradient program from $0 \%$ to $50 \%$ of sol. B in sol. A for $30 \mathrm{~min}$ was employed at a flow rate of $1.0 \mathrm{ml} / \mathrm{min}$ (analytical) or 15 $\mathrm{ml} / \mathrm{min}$ (preparative). The wavelength of the detector was set at $440 \mathrm{~nm}$. Eluants from the preparative HPLC column were collected when the relevant zones were eluted, evaporated at $40^{\circ} \mathrm{C}$, and the residues were used as standard pigments for the MEKC analysis. Isocratic elution was performed for geniposide and gardenoside with distilled water: acetonitrile (80:
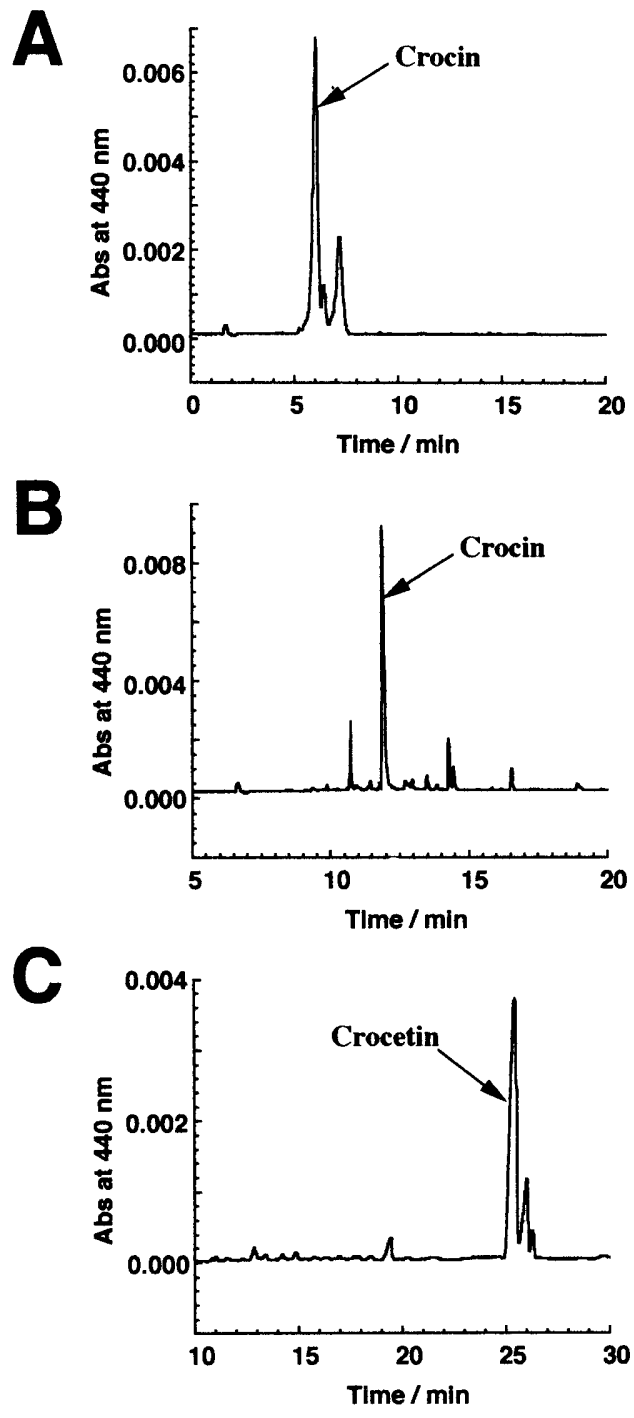

Fig. 2. Separation of the pigments $A$ and $B$ by MEKC with SDS. A) sample, pigment A (containing mainly crocin); capillary, $50 \mu \mathrm{m}$ i.d. $\times 50 \mathrm{~cm}$; running solution, $50 \mathrm{~mm}$ phosphate buffer at $\mathrm{pH} 7.0$ containing $20 \mathrm{~mm}$ SDS; applied voltage, $15 \mathrm{kV}$; temperature, $20^{\circ} \mathrm{C}$; detection, $440 \mathrm{~nm}$. B) running solution, 20 $\mathrm{mm}$ SDS solution in $50 \mathrm{~mm}$ phosphate buffer at $\mathrm{pH} 7.0$ containing $20 \%$ acetonitorile; the sample and other conditions are the same as those in $\mathrm{A}$. C) sample, pigment B (containing mainly crocetin); the other conditions are the same as those in B.

20) at a flow rate of $1.0 \mathrm{ml} / \mathrm{min}$. The wavelength of the detector was set at $237 \mathrm{~nm}$.

$M E K C$ separation For the analysis of yellow pigments, two running solutions were employed: a $20 \mathrm{~mm}$ SDS solution in $50 \mathrm{~mm}$ phosphate buffer ( $\mathrm{pH} 7.0$ ) containing $20 \%$ acetonitrile and a 2.0\% BBMA in $20 \mathrm{mM}$ ammonium formate buffer ( $\mathrm{pH} 7.0$ ) containing 10\% methanol. A potential of 15 $\mathrm{kV}$ was applied, the detection was performed by measuring absorbance at $440 \mathrm{~nm}$, and the capillary temperature was $20^{\circ} \mathrm{C}$. For the analysis of geniposide and gardenoside, a 20 mM SDS solution in $30 \mathrm{~mm}$ borate buffer ( $\mathrm{pH} 8.5)$ was used with the detection at $240 \mathrm{~nm}$. The applied voltage and temperture were the same as above. The samples were dissolved in distilled water, and the solution was passed through a disposable hydrophilic membrane filter HP-20 $(0.45-\mu \mathrm{m}$ pore size; Advantec Toyo) prior to analysis. The 
filtrates were injected by pressure at $350 \mathrm{mbar}$ for $1.0 \mathrm{~s}$. The capillary was rinsed with a $1.0 \mathrm{M} \mathrm{NaOH}$ solution for $120 \mathrm{~s}$ and with distilled water for $120 \mathrm{~s}$ before each run.

\section{Results and Discussion}

$M E K C$ analysis of yellow pigments In MEKC, an ionic micellar solution is used as the separation solution. Solutes that are totally solubilized by the micelles elute at the migration time of the micelle, $t_{\mathrm{mc}}$. Hydrophilic solutes that are insoluble in the micelles elute at the migration time of the bulk solution, $t_{0}$, which is dependent on the electroosmotic flow rate. All solutes must elute between $t_{0}$ and $t_{\mathrm{mc}}$, provided they are electrically neutral. The resolution becomes better when the migration time window, $t_{\mathrm{mc}} / t_{0}$, is wider. Addition of a small volume of acetonitrile to the running solution usually causes better resolution, because of the extention of the migration time window due to the decrease in the electroosmotic flow rate.

MEKC with an SDS solution in a phosphate buffer $(\mathrm{pH}$ 7.0) was not very successful in separating pigment $A$ as shown in Fig. 2A. As shown in Fig. 2B, 20\% addition of acetonitrile to the running solution gave better resolution. Pigment $\mathrm{B}$ was also successfully separated by MEKC with SDS containing acetonitrile (Fig. 2C). Each main peak in Figs. 2B and 2C was presumed to be crocin and crocetin, respectively. Also, a 2.0\% BBMA solution in $20 \mathrm{~mm}$ ammonium formate $(\mathrm{pH} 7.0)$ gave successful separation of pigment $A$ and $B$ as shown in Figs. $3 \mathrm{~A}$ and $3 \mathrm{~B}$, but the migration order changed between crocin and crocetin. The change can be explained in terms of different selectivity between the two surfactants.

In order to check the reproducibility of the separation of gardenia yellow pigments by MEKC with the SDS solution containing acetonitrile, pigment $\mathrm{A}$ was repeatedly analyzed fifteen times. No significant difference in the separation pattern and migration time was observed among the repeated runs for pigment $\mathrm{A}$. Thus, we found that MEKC with SDS containing acetonitrile was the best method for the analysis of gardenia yellow pigments.

Each yellow pigment, crocin and crocetin, prepared by preparative HPLC gave a single peak and was used as a
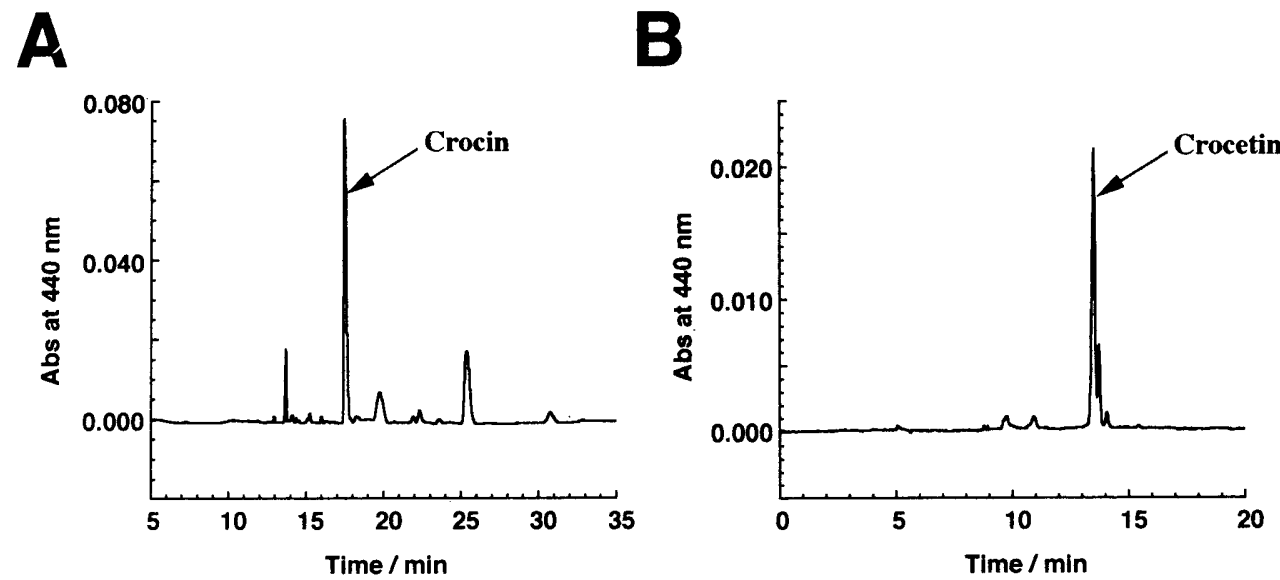

Fig. 3. Separation of pigments $A$ and $B$ by MEKC with BBMA. A) sample, pigment A; running solution, 20 mm ammonium formate buffer at pH 7.0 containing 2.0\% BBMA; The other conditions are the same as those in Fig. 2A. B)sample, pigment B; the other conditions are the same as those in A.
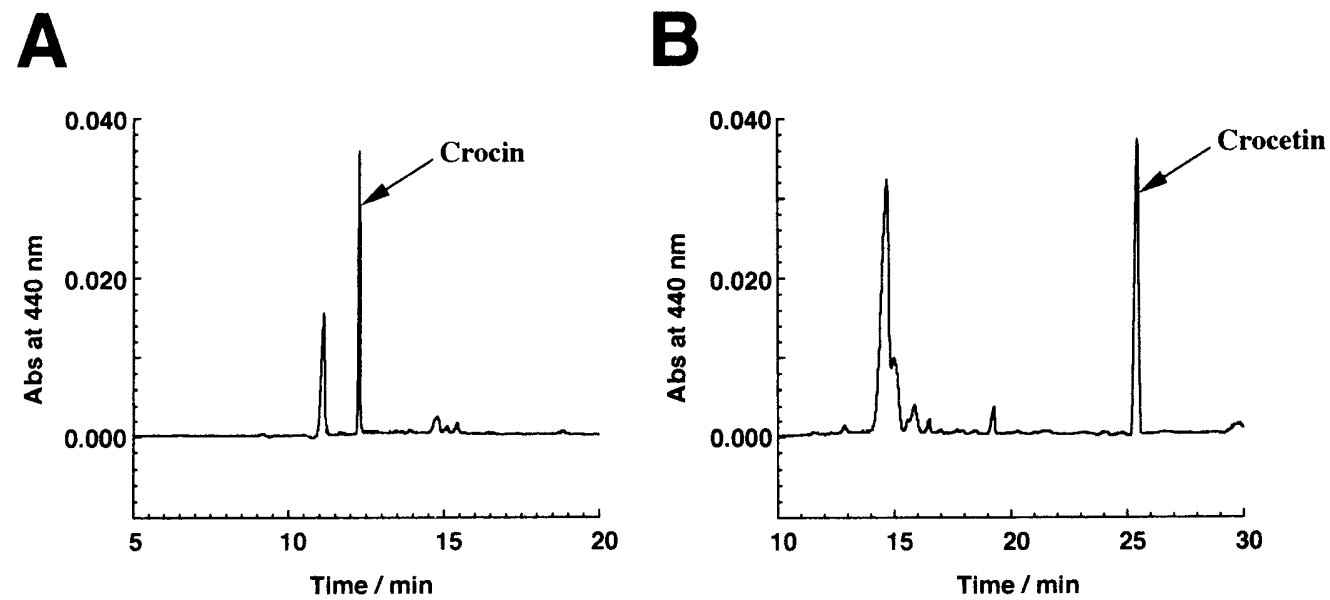

Fig. 4. Separation of yellow pigments extracted from food samples by MEKC with SDS. A, pigment A extracted from candies; B, pigment B extracted from noodles. The conditions are the same as those in Fig. 2B. 
Table 1. MEKC analytical results for the yellow pigment extracted from food samples.

\begin{tabular}{|c|c|c|c|c|c|}
\hline \multirow[b]{2}{*}{ Sample } & \multirow{2}{*}{$\begin{array}{c}n \\
\text { (Intra- } \\
\text { day) }\end{array}$} & \multicolumn{2}{|c|}{ Migration time } & \multicolumn{2}{|c|}{ Determination } \\
\hline & & $\begin{array}{l}\text { Mean } \\
(\mathrm{min})\end{array}$ & $\begin{array}{l}\text { C.V. } \\
\text { (\%) }\end{array}$ & $\begin{array}{c}\text { Mean } \\
\left(\mu \mathrm{g} \mathrm{g}^{-1}\right)\end{array}$ & $\begin{array}{l}\text { C.V. } \\
(\%)\end{array}$ \\
\hline \multicolumn{6}{|l|}{ Standard } \\
\hline Crocin pigment $^{a)}$ & 3 & 12.28 & 0.38 & 64 & 1.57 \\
\hline Crocetin pigment $^{b)}$ & 3 & 25.18 & 0.42 & 88 & 2.06 \\
\hline \multicolumn{6}{|c|}{ Yellow pigment extracted from food samples } \\
\hline From candies ${ }^{a)}$ & 3 & 12.22 & 0.48 & 590 & 2.54 \\
\hline From noodles ${ }^{b)}$ & 3 & 25.24 & 0.64 & 67 & 2.38 \\
\hline
\end{tabular}

${ }^{a)}$ Crocin is mainly contained.

${ }^{b)}$ Crocetin is mainly contained.
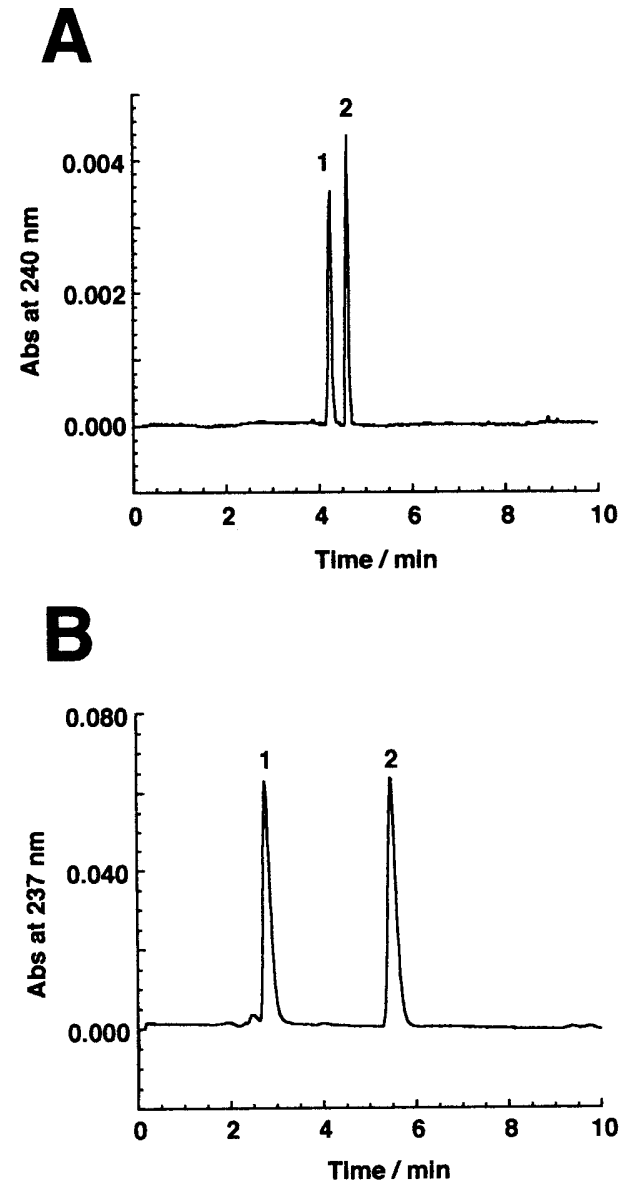

Fig. 5. Separation of geniposide and gardenoside by MEKC and HPLC. A) MEKC separation; running solution, $20 \mathrm{~mm}$ SDS solution in $30 \mathrm{~mm}$ borate buffer at $\mathrm{pH} 8.5$; detection, $240 \mathrm{~nm}$. B) HPLC separation; mobile phase, distilled water:acetonitorile $=80: 20$; flow rate, $1.0 \mathrm{ml} / \mathrm{min}$; detection, $237 \mathrm{~nm}$. The other conditions are the same as those in Fig. 2A. Peak identification, $1=$ Gardenoside; $2=$ Geniposide.

standard for the MEKC analysis. The standard curves for calibration obtained with these standards from 5 to $100 \mu \mathrm{g} /$ g showed straight lines (crocin: $r=0.997$; crocetin: $r=0.996$ ), the detection limits for both yellow pigments being $0.2 \mu \mathrm{g} / \mathrm{g}$. Crocin and crocetin contents in pigments $\mathrm{A}$ and $\mathrm{B}$ were determined with the standard curve.

Analysis of the pigments extracted from food samples
Table 2. MEKC determination of the gardenoside and geniposide extracted from gardenia fruit samples.

\begin{tabular}{lccc}
\hline \multirow{2}{*}{ Habitat } & \multicolumn{2}{c}{ Determination $\left(\mathrm{mg} \mathrm{g}^{-1}\right)$} & $\begin{array}{c}\text { Gardenoside/ } \\
\text { Geniposide }\end{array}$ \\
\cline { 2 - 3 } & Gardenoside & Geniposide & 0.46 \\
Sichuan (China) & 27.2 & 59.1 & 0.50 \\
Guangxi (China) & 28.0 & 56.2 & 0.38 \\
Hubei (China) & 28.7 & 76.1 & 0.39 \\
Shanghai (China) & 17.2 & 44.5 & 0.39 \\
Zhejiang (China) & 17.2 & 44.5 & 0.75 \\
Taiwan & 30.8 & 41.2 & 0.88 \\
Korea & 27.2 & 30.8 & \\
\hline
\end{tabular}

Crocin and crocetin were successfully extracted from candies and noodles by the SPE method. The recovery of each pigment by SPE was found to be $82 \%$, which was determined by a decrease in the absorbance of each aqueous solution of the original yellow pigment by SPE. These yellow pigments were analyzed by MEKC with the SDS solution containing acetonitrile. Figure 4A shows the electropherogram of the pigments extracted from candies. The major peak was easily assigned to crocin by the migration time of crocin given in Fig. 2B. Figure 4B shows the MEKC separation of the pigments extracted from noodles, and the peak at $25 \mathrm{~min}$ was similarly identified as that of crocetin (see Fig. 2C). The coefficient of variation (CV) of the migration time was less than $0.7 \%$, and that for the determination was less than $2.6 \%$ (Table 1).

$M E K C$ analysis of geniposide and gardenoside For the separation of geniposide and gardenoside, MEKC using a $20 \mathrm{~mm}$ SDS in $30 \mathrm{~mm}$ borate buffer ( $\mathrm{pH} \mathrm{8.5)}$ was successful as shown in Fig. 5A. The separation by a linear gradient HPLC was also successful as shown in Fig. 5B. Compared to the HPLC method, the MEKC method is advantageous due to its low running cost and shorter analysis times.

Standard curves obtained with geniposide and gardenoside from 1 to $50 \mu \mathrm{g} / \mathrm{g}$ showed straight lines (geniposide: $r=0.998$; gardenoside: $r=0.997$ ), the detection limits for both components being $0.05 \mu \mathrm{g} / \mathrm{g}$. The geniposide and the gardenoside in gardenia fruits were determined with these standard curves.

Analysis of the geniposide and gardenoside extracted from gardenia fruit samples The geniposide and gardenoside in gardenia fruits were determined by the MEKC method. Table 2 shows the contents of geniposide and gardenoside in gardenia fruits from several habitats. Zhejiang (China)-grown gardenia fruits contained about $46 \mathrm{mg} / \mathrm{g}$ geniposide and $16 \mathrm{mg} / \mathrm{g}$ gardenoside (gardenoside/geniposide $=0.35$ ); however, Korea-grown gardenia fruits contained about $31 \mathrm{mg} / \mathrm{g}$ geniposide and $27 \mathrm{mg} / \mathrm{g}$ gardenoside (gardenoside/geniposide $=0.88$ ). Hubei (China)-grown gardenia fruits contained the greatest amount of the geniposide, and Taiwan-grown gardenia fruits had the highest content of the gardenoside.

In conclusion, MEKC was found to be a useful technique for analyzing crocin, crocetin, geniposide and gardenoside. The amount of the sample required for the analysis was very small, and the repeatability of migration times and quantitation was high even for real samples, probably due to the absence of any packing material inside the capillary. Another 
advantage of the $\mathrm{CE}$ method is its low running cost. This MEKC method can be applied to quality control analysis in food additives. The CE method will also be widely applicable to the analysis of different food additives.

\section{References}

Ichi, T., Higashimura, Y., Katayama, T., Koda, T., Shimizu, T. and Tada, M. (1995a). Analysis of crocetin derivatives from gardenia fruits. Nippon Shokuhin Kagaku Kogaku Kaishi, 42, 776-783 (in Japanese).

Ichi, T., Higashimura, Y., Katayama, T., Koda, T., Shimizu, T. and Tada, M. (1995b). Food chemical properties of crocetin derivatives in gardenia yellow color. Nippon Shokuhin Kagaku Kogaku Kaishi, 42, 784-789 (in Japanese).

Ichi, T., Higashimura, Y., Katayama, T., Koda, T. and Tada, M. (1995c). Determination of a food colorant, gardenia yellow pigment, in processed foods with a high performance liquid chromatography/photodiode-array detector/mass spectrometer system. Shokuhin Eiseigaku Zasshi, 36, 482-489 (in Japanese).

Kamikura, M. and Nakazato, K. (1984). Chromatographic separation and determination of geniposide in commercial gardenia fruit extract color. Shokuhin Eiseigaku Zasshi, 25, 517-524 (in Japanese).

Kamikura, M. and Nakazato, K. (1985). Natural yellow colors extracted from gardenia fruits and colors found in commercial gardenia fruit extract color. Shokuhin Eiseigaku Zasshi, 21, 207213 (in Japanese).

Nishi, H., Tsumagari, N., Kakimoto, T. and Terabe, S. (1989). Separation of water-soluble vitamines by micellar electrokinetic chromatography. J. Chromatogr., 465, 331-343.

Nishizawa, M., Chonan, T., Sekijo, I. and Sugii, T. (1983). Analysis of anatto extract and gardenia yellow dye in foods and dye preparations. Hokkaido Eisei Kenkyushoho, 33, 28-34 (in Japanese).

Noda, N., Yamada, S., Hayakawa, J. and Uno, K. (1983). Analysis of natural dye in foods. Determination of natural yellow dye from the fruits of gardenia by detecting geniposide. Eisei Kagaku, 29, 7-12 (in Japanese).

Ogiwara, T., Nozawa, T., Hirata, K., Amekawa, E., Oishi, M. and Seki, H. (1980). Detection of water-soluble natural dyes by a polyamide batch method. Shokuhin Eiseigaku Zasshi, 21, 207-213 (in Japanese).

Terabe, S., Otsuka, K. and Ando, T. (1985). Electrokinetic chromatography with micellar solution and open-tubular capillary. Anal. Chem., 57, 834-841.

Terabe, S., Otsuka, K., Ichikawa, K., Tsuchiya, A. and Ando, T. (1984). Electrokinetic separations with micellar solutions and opentubular capillaries. Anal. Chem., 56, 111-113.

Touyama, R., Takeda, Y., Inoue, K., Kawamura, I., Yatsuzuka, M., Ikumoto, T., Shingu, T., Yokoi, T. and Inoue, H. (1994). Studies on the blue pigments produced from genipin and methylamine. I. structures of the brownish-red pigments, intermediates leading to the blue pigments. Chem. Pharm. Bull., 42, 668-673.

Watanabe, T., Hasegawa, N., Y amamoto, A., Nagai, S. and Terabe, S. (1996). Capillary electrophoresis of anthraquinone pigments for food. Bunseki Kagaku, 45, 765-770 (in Japanese).

Watanabe, T., Hasegawa, N., Yamamoto, A., Nagai, S. and Terabe, S. (1997a). Separation and determination of yellow and red safflower pigments in food by capillary electrophoresis. Biosci. Biotech. Biochem., 61, 1179-1183.

Watanabe, T., Yamamoto, A., Nagai, S. and Terabe, S. (1997b). Separation and determination of monascus yellow pigments for food by micellar electrokinetic chromatography. Anal. Sci., 13, 571575 . 